\title{
Cancer/testis antigen NY-SAR-35 enhances cell proliferation, migration, and invasion
}

\author{
MYUNG-HA SONG ${ }^{1}$, YE-RIN KIM ${ }^{1}$, JUN-WON LEE $^{3}$, CHANG-HUN LEE $^{2}$ and SANG-YULL LEE ${ }^{1}$ \\ Departments of ${ }^{1}$ Biochemistry and ${ }^{2}$ Pathology, School of Medicine, Pusan National University, Yangsan, \\ Gyeongsangnam-do 626-870; ${ }^{3}$ Department of Life Science and Genetic Engineering, \\ Paichai University, Daejeon 302-735, Republic of Korea
}

Received October 5, 2015; Accepted November 6, 2015

DOI: $10.3892 /$ ijo.2015.3264

\begin{abstract}
The cancer/testis antigen NY-SAR-35 is aberrantly expressed in various cancer tissues and cancer cell lines but not in normal tissues except for the testis. A previous study demonstrated that the expression of NY-SAR-35 is activated by hypomethylation in cancer cells. However, the functions of this antigen remain unexplored. In the present study, we investigated the role of NY-SAR-35 in human embryonic kidney (HEK) 293 cells using exogenous expression system of the gene. NY-SAR-35 was predominantly expressed at the cytoplasm and was mainly observed in spermatogonia and spermatocytes. Expression of NY-SAR-35 in stable HEK293 transfectant clones was 2-fold higher than the control cells promoting cell growth and proliferation. NY-SAR-35 overexpression also enhanced cell migration and invasion $\sim 2$-fold and 4-fold more than the control, respectively. In contrast, small interfering RNA-mediated knockdown of NY-SAR-35 suppressed cell proliferation, migration, and invasion in HEK293 stable transfectants. We concluded that NY-SAR-35 as a cancer/testis antigen enhanced cell proliferation and invasion.
\end{abstract}

\section{Introduction}

Cancer/testis (CT) antigens are a category of tumor-associated antigens expressed in a proportion of many different types of cancers, but not in normal tissues, except for testis and placenta (1-4). Also, CT antigens are frequently mutated in cancers, and many CT antigens were identified by using sera of cancer patients $(3,5-9)$, implying they are highly antigenic. CT antigens are classified into two categories based on the chromosomal location (3). CT-X antigens are located on the $\mathrm{X}$ chromosome, are often members of gene families, and

Correspondence to: Professor Sang-Yull Lee, Department of Biochemistry, School of Medicine, Pusan National University, Beomeo-ri, Mulgeum-eup, Yangsan-si, Gyeongsangnam-do 626-870, Republic of Korea

E-mail: sangyull@pusan.ac.kr

Key words: cancer/testis antigen, NY-SAR-35, proliferation, migration, invasion represent more than half of all CT antigens identified. In contrast, non-X CT antigens are distributed throughout the genome and mostly single-copy genes. To date, >200 CT antigens, including MAGE, NY-ESO-1, GAGE, AKAP3, SSX, and LAGE, have been identified, but the function of most is largely unknown $(3,10-12)$.

Recent studies indicated that members of CT antigens are likely involved in cell cycle regulation, transcriptional control, cell survival, and apoptosis (5,13-21). CT45A1, a member of cancer/testis antigen-45 family (CT45), functions as a novel proto-oncogene by upregulating various oncogenic and metastatic genes and activating ERK and CREB signaling pathways to trigger oncogenesis and metastasis (17). Another study on functions of a CT antigen reported that the growth promoting and survival properties of SSX are mediated in part through modulation of MAPK/Erk and Wnt signaling pathways (22).

Among CT antigens, NY-SAR-35 was previously identified by SEREX analysis from human sarcoma patients. The NY-SAR-35 gene is located on chromosome Xq28 like several other CT antigens and not a member of a multigene family. In addition, the putative NY-SAR-35 protein is expected to have a signal peptide domain, a transmembrane domain, and a cystein-rich trefoil/P-domain (23). Despite the expression of NY-SAR-35 in various malignancies, it is expressed with low frequency or not expressed in some cancer cells $(3,24)$. In our previous study, treatment with the DNA demethylating agent can also lead to an increase in the absent or downregulated expression of NY-SAR-35, and transcriptional silencing of NY-SAR-35 is caused by hypermethylation of its promoter (25). This finding proposed hypomethylation of $\mathrm{CpG}$ islands within the promoter region be a primary mechanism for transcriptional activation of NY-SAR-35 gene in cancer. However, the functions of this antigen remain unexplored.

In the present study, the functional effect of NY-SAR-35 on cell growth, proliferation, and invasion was assessed by an in vitro cell culture system in HEK293 cell line.

\section{Materials and methods}

Cell culture. The human embryonic kidney (HEK) 293 and the murine fibroblast cell line NIH 3T3 cells were cultured in DMEM (Sigma-Aldrich, St. Louis, MO, USA) supplemented with $10 \%$ (v/v) FBS, 2 mM L-glutamine, $100 \mathrm{U} / \mathrm{ml}$ penicillin, 
and $100 \mu \mathrm{g} / \mathrm{ml}$ streptomycin. The cells were obtained from American Type Culture Collection (ATCC) and cultured at $37^{\circ} \mathrm{C}$ in a humidified atmosphere containing $5 \% \mathrm{CO}_{2}$. The study was conducted under an approved protocol from the Ethics Committee of Pusan National University.

RNA isolation and RT-PCR. Total RNA was extracted from cells by using the RNeasy mini kit (Qiagen, Hilden, Germany). The total RNA of cDNA was synthesized from $1 \mu \mathrm{g}$ total RNA by using M-MLV reverse transcriptase (Promega, Fitchburg, WI, USA). Primers used were NY-SAR-35-forward (5'-CTTGGTGCGA TCAGCCTTAT-3') and NY-SAR-35reverse (5'-TTGATGCA TGAAAACAGAAC-3'). PCR amplification was carried out as follows: denaturation for $5 \mathrm{~min}$ at $94^{\circ} \mathrm{C}$, then 35 cycles consisting of $30 \mathrm{sec}$ at $94^{\circ} \mathrm{C}$, $30 \mathrm{sec}$ at $55^{\circ} \mathrm{C}$, and $1 \mathrm{~min}$ at $72^{\circ} \mathrm{C}$ and then $10 \mathrm{~min}$ at $72^{\circ} \mathrm{C}$ for final extension. PCR products were analyzed by agarose gel electrophoresis and visualized by ethidium bromide.

Western blot analysis. Cells were lysed in lysis buffer and cellular debris was removed by centrifugation at 15,000 rpm for $10 \mathrm{~min}$. Proteins were separated by sodium dodecyl sulfate-polyacrylamide gel electrophoresis, transferred onto nitrocellulose membranes (Hybond-ECL, GE Healthcare, UK), blocked with 5\% skim milk and incubated with anti-V5 or anti-His (Life Technologies, Carlsbad, CA, USA) and anti- $\beta$ actin antibodies (Sigma-Aldrich). Membranes were then incubated with horseradish peroxidase-conjugated goat antimouse-IgG secondary antibodies for $1 \mathrm{~h}$ at room temperature and detected by enhanced chemiluminescence (Perkin-Elmer Life Science, MD, USA).

Generation of recombinant NY-SAR-35 protein. The open reading frame (ORF) cDNA inserts of NY-SAR-35 (AY211917) were selected to generate His-tagged NY-SAR-35 protein. The primers for the partial protein were GGAATTCCATATGCT GCCCAACAGT (forward) and CCGCTCGAGCATTTGCA TCATCT (reverse) containing NdeI and XhoI restriction enzyme sites. PCR products were subcloned into the pET21a expression plasmid containing a polyhistidine-tag. The recombinant NY-SAR-35 fusion protein was induced by isopropyl $\beta$-D-thiogalactoside (IPTG) with a final concentration of $0.4 \mathrm{mM}$ at $37^{\circ} \mathrm{C}$. Affinity chromatography using Ni-NTA agarose (Qiagen) was performed to purify NY-SAR-35 recombinant protein. The purified recombinant protein was determined by SDS-PAGE and Coomassie Blue staining.

Production and purification of polyclonal NY-SAR-35 antibodies. Antibodies against NY-SAR-35 were raised in a naïve New Zealand white rabbit immunized subcutaneously with $200 \mu \mathrm{g}$ of recombinant NY-SAR-35 protein. Three booster injections were given using the same antigen, the first booster three weeks after the first immunization and then each week. The rabbit was then sacrificed and the crude antiserum was collected. Especially for the isolation of NY-SAR-35 purified antibodies, affinity purification was performed using cyanogen bromide (CNBr)-activated sepharose beads (GE Healthcare). Normally, coupling to beads was achieved at $\mathrm{pH} 8.3$, while elution occurred at $\mathrm{pH}$ 2.8. Neutralization by adding Tris buffer with $\mathrm{pH} 8.8$ was performed as soon as possible after elution to prevent damage of the antibody.

Construction of stable and transient cell lines. To generate stably transfected cell lines, ORF of NY-SAR-35 including 5'-untranslated region was cloned into pcDNA3.1/V5-HisA vector which has C-terminal fusion tag (V5 and 6X His epitopes) containing EcoRI and XhoI sites. Then, HEK293 cells ( $1 \times 10^{6}$ cells in $60-\mathrm{mm}$ dishes) were transfected with $4 \mu \mathrm{g}$ of cloned pcDNA3.1/V5-HisA-NY-SAR-35 using Lipofectamine LTX (Life Technologies). Transfected cells were selected in medium containing G418 (1 mg/ml). The selected clones were maintained in medium containing G418 $(0.3 \mathrm{mg} / \mathrm{ml})$.

For transient transfection, the cDNA of complete coding region of NY-SAR-35 was generated by PCR amplification using primers containing XhoI and PstI restriction sites. The PCR products were then inserted in-frame within the pEGFP-N1 vector. The NIH 3T3 cell was transfected with cloned pEGFP-N1-NY-SAR-35.

siRNA transfection. The small interefing RNA (siRNA) used for the targeted silencing of NY-SAR-35 (5'-GCCUUAUCCUGGU AUGUCUTT-3') was purchased from Qiagen. In brief, cells $\left(2 \times 10^{5}\right.$ cells $\left./ \mathrm{ml}\right)$ were seeded on 6 -well plates and transfected with $200 \mathrm{nM}$ of siRNA using oligofectamine reagent, according to the manufacturer's instructions (Invitrogen, Carlsbad, CA, USA). Cells were then incubated at $37^{\circ} \mathrm{C}$ for $4 \mathrm{~h}$ in serum-free media. The transfected cells were collected after $48 \mathrm{~h}$ and NY-SAR-35 expression levels in the cells were determined using RT-PCR.

Immunohistochemistry. Staining was conducted with the peroxidase-based EnVision Detection kit (DakoCytomation, USA) by following the user manual. Briefly, tissue sections were deparaffinized and hydrated in xylene and graded alcohol series. Then, the sections were incubated with the purified NY-SAR-35 antibody for $1 \mathrm{~h}$ at room temperature. After incubation with EnVision solution, the 3, 3'-diaminobenzidine (DAB) substrate was added followed by hematoxylin counterstaining and mounting.

Cell counting. Cell numbers were determined as follows: cells (5x105/90-mm culture plate) were cultured for 4 days in standard culture medium supplemented with 1 or $10 \%$ FBS. After incubation for 4 days, adherent and vital cells were counted in a trypan blue exclusion assay.

Bromodeoxyuridine (BrdU) incorporation assay. Cell proliferation was measured by 5-bromo-2'-deoxyuridine (BrdU) incorporation using the Cell Proliferation ELISA, BrdU kit (Roche Diagnostics, Germany) according to the manufacturer's instructions. In brief, cells were grown in 96-well plates and labeled with BrdU for $2 \mathrm{~h}$. Subsequently, anti-BrdU-POD Fab fragments and substrates were added to the medium. The optical density (OD) was determined at $450 \mathrm{~nm}$ using an ELISA reader (Bio-Tek Instruments, VT, USA).

Immunofluorescent staining. Cells grown on coverglassbottom confocal dish were fixed with $4 \%$ paraformaldehyde. Then, cells were blocked in $3 \% \mathrm{FBS} / \mathrm{PBS}$ and incubated 

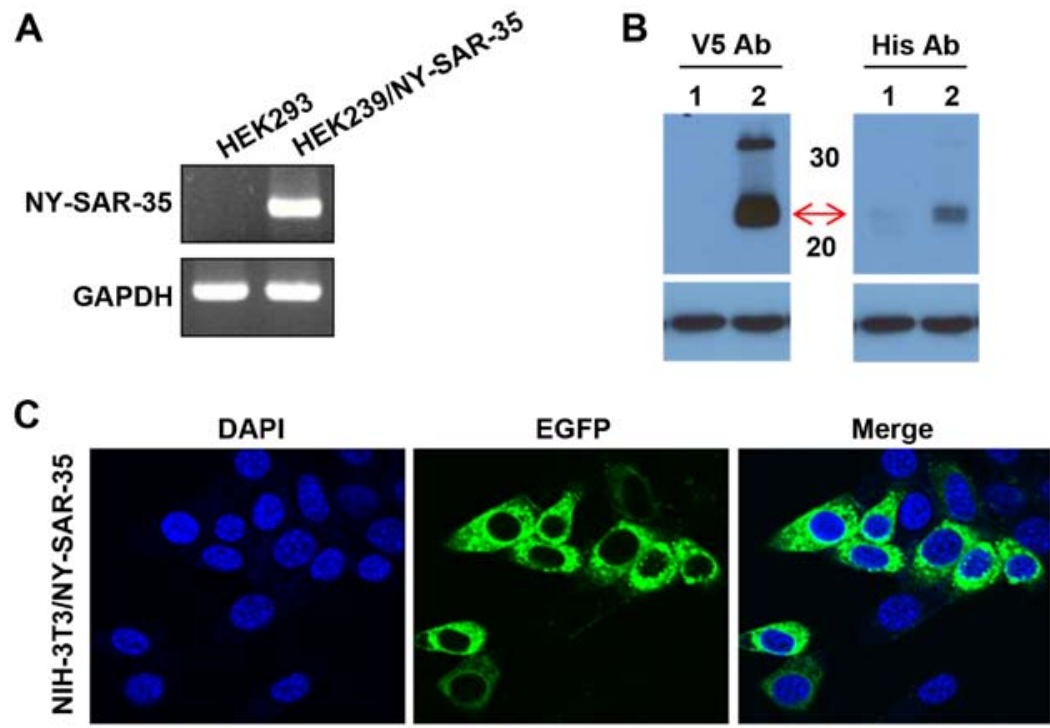

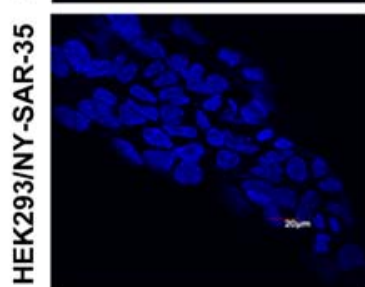

DAPI

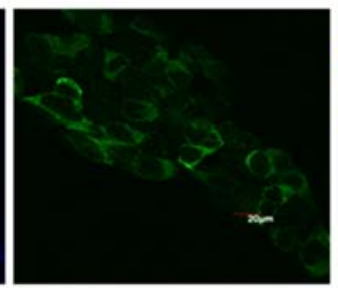

V5-FITC

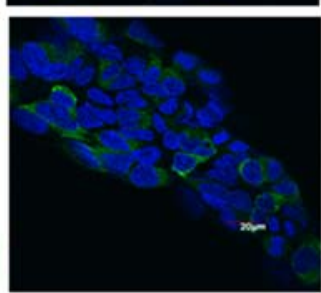

Merge

\section{D}
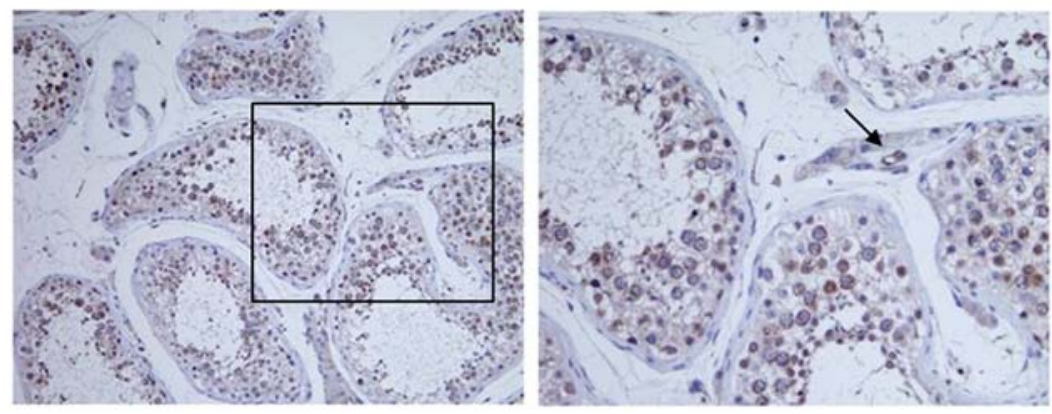

Figure 1. Expression and localization of NY-SAR-35 in HEK293 cells. (A) RT-PCR of the transfected NY-SAR-35 gene using NY-SAR-35 specific primer set. HEK293 cells are the negative control. (B) Western blot analysis of HEK293 cells (lane 1) and HEK293 cells stably expressing NY-SAR-35 (lane 2). Numbers in the middle show the positions of MW markers ( $\mathrm{kDa}$ ) and the arrow indicates MW of detected bands. (C) Co-localization study of the NY-SAR-35 and EGFP or V5 fragments with immunofluorescence microscope in transfected NIH 3T3 and HEK293 cells, respectively. (D) Pattern of NY-SAR-35 expression in testis tissue. Low magnification (x200, left) image and higher magnification (x400, right) image indicated by the box in the left panel are shown. NY-SAR-35 was expressed as a cytoplasmic protein in spermatogonia and primary and/or secondary spermatocytes. The arrowhead in the right panel indicates positive for interstitial blood vessel.

with anti-PCNA (proliferating cell nuclear antigen) or Ki-67 antibody. The cells were washed three times with PBS, incubated with FITC-coupled secondary antibodies. Images were captured using confocal microscopy (Olympus).

Cell migration and invasion assay. To measure the cell migration activity, transwell chamber assays were performed using a transwell plate (Becton-Dickinson, MA, USA). The lower surface of the filters was coated with $1 \%$ gelatin. Cells were resuspended in serum-free medium and then added to the upper chamber at a density of $5 \times 10^{4}$ cells/insert. The medium containing $10 \%$ FBS was added to the lower chamber. After the cells were incubated at $37^{\circ} \mathrm{C}$ for $18 \mathrm{~h}$, the number of cells that migrated to the lower side of the upper chamber was counted by staining with crystal violet. The invasive potentials of the cells were analyzed using a Matrigel-coated modified Boyden chamber (Becton-Dickinson). The medium containing $10 \%$ FBS was added to the lower chamber as an attractant. The incubations of the cells were continued for $24 \mathrm{~h}$. Inserts were then stained with crystal violet and the number of invaded cells was counted.

\section{Results}

NY-SAR-35 protein is located in the cytoplasm. To investigate the role of NY-SAR-35 gene, NY-SAR-35-transfected HEK293 cells were established with pcDNA3.1/V5-HisA-NY-SAR-35 vector and named HEK293/NY-SAR-35. Expression of NY-SAR-35 in HEK293/NY-SAR-35 cells was confirmed with RT-PCR (Fig. 1A) and western blot analysis (Fig. 1B). In 
A

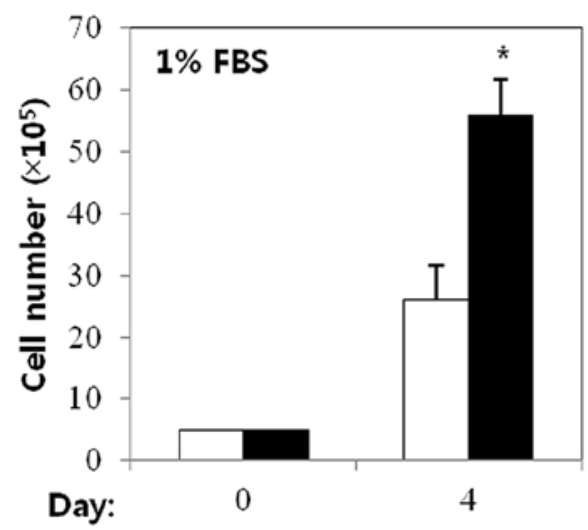

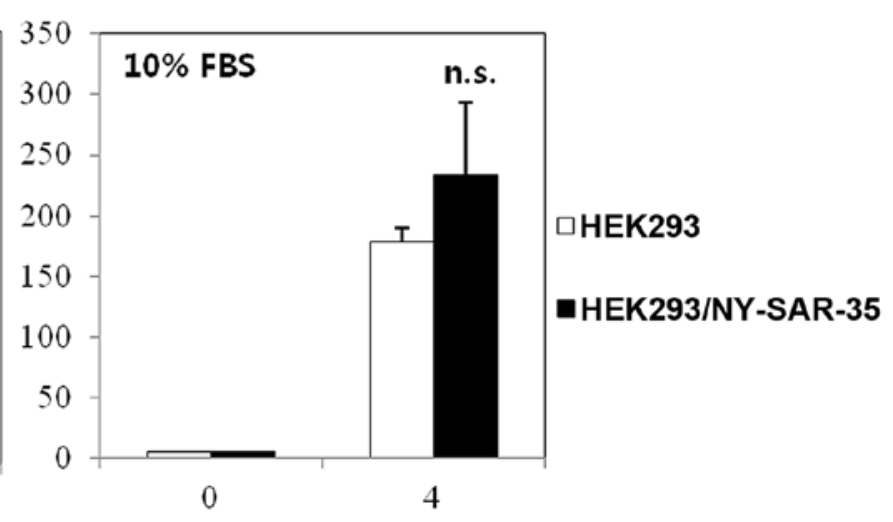

B

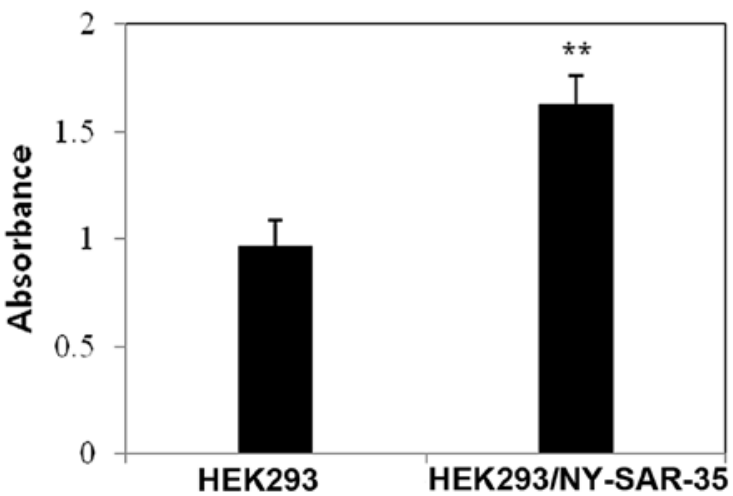

C
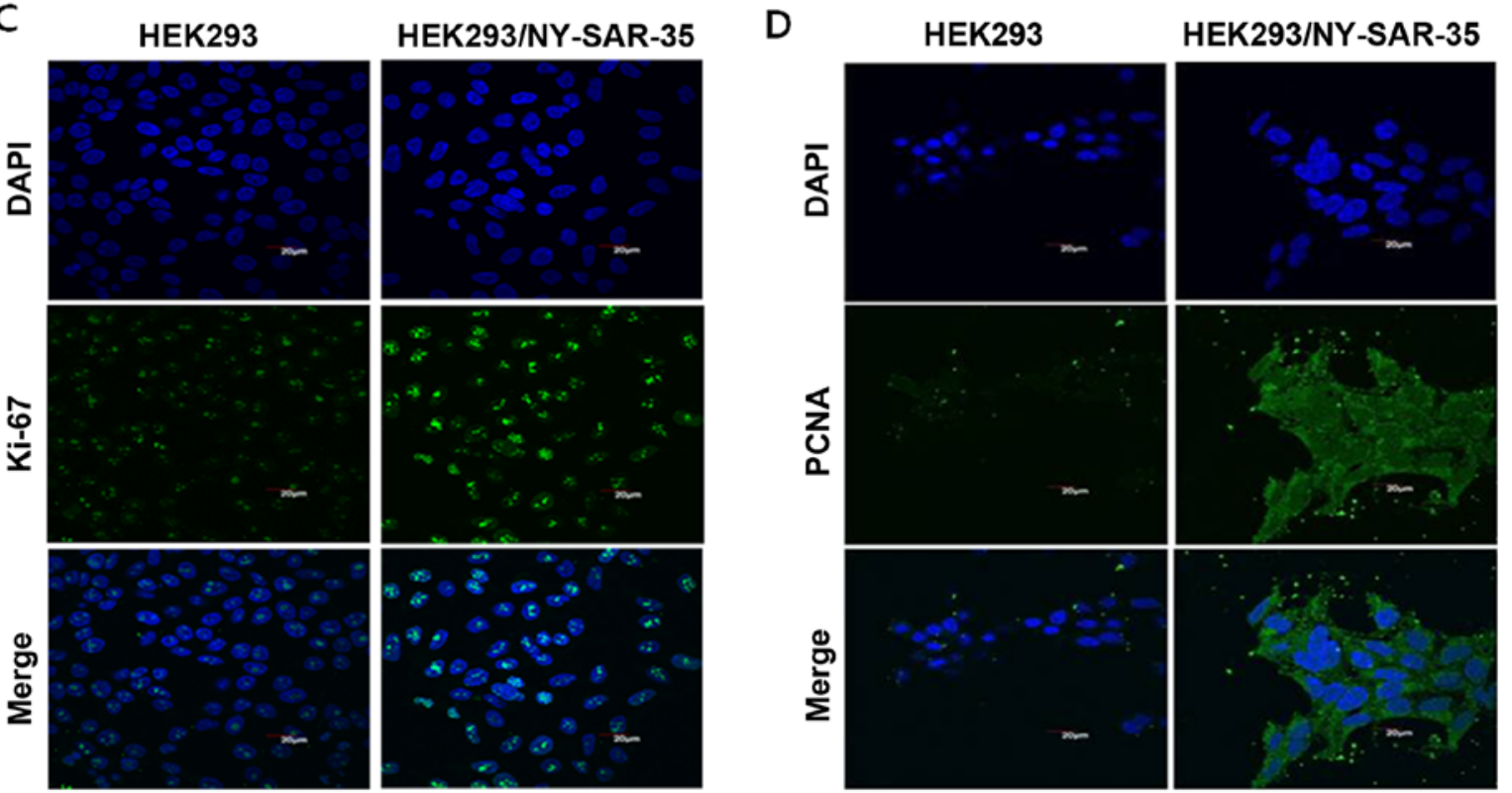

Figure 2. Effects of NY-SAR-35 expression on cell growth and DNA synthesis of HEK293 cells. (A) HEK293 (white bar) and HEK293/NY-SAR-35 (black bar) cells were cultured in medium containing 1 or $10 \%$ FBS, and adherent and vital cells were counted at day 4. (B) In vitro effects of NY-SAR-35 on cell proliferation were measured by the incorporation of BrdU into synthesized DNA. Absorbance is measured at a wavelength of $450 \mathrm{~nm}$ with a reference wavelength of $690 \mathrm{~nm}$. Cells were phenotyped by immunofluorescence analysis after staining for proliferation markers, Ki-67 (C) and PCNA (D). HEK293 cells are the negative control (magnification, $\mathrm{x} 600$ ). Bars represent mean values, n.s., no significant difference; ${ }^{*} \mathrm{P}<0.05 ;{ }^{* * *} \mathrm{P}<0.01$.

addition, immunofluorescent staining with EGFP or V5-FITC showed that NY-SAR-35 was predominantly observed at the cytoplasm in NIH3T3/NY-SAR-35 and HEK293/NY-SAR-35 cells, respectively (Fig. 1C).

Using purified antibody, the expression of NY-SAR-35 protein in normal testis tissue was analyzed using immunohistochemical techniques. Expression of NY-SAR-35 was mainly observed in spermatogonia and spermatocytes, as cytoplasmic proteins. In addition, a positive reaction was seen in vascular endothelial cells, but not Leydig cells in interstitium (Fig. 1D).

NY-SAR-35 enhances cell growth and proliferation in HEK293 cells. Because NY-SAR-35 protein seemed to be involved in 
A

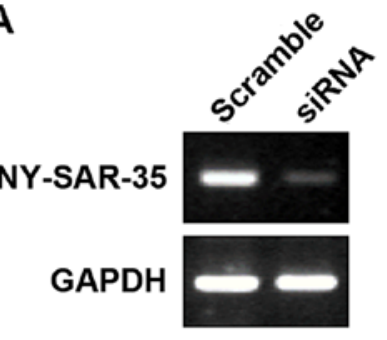

B
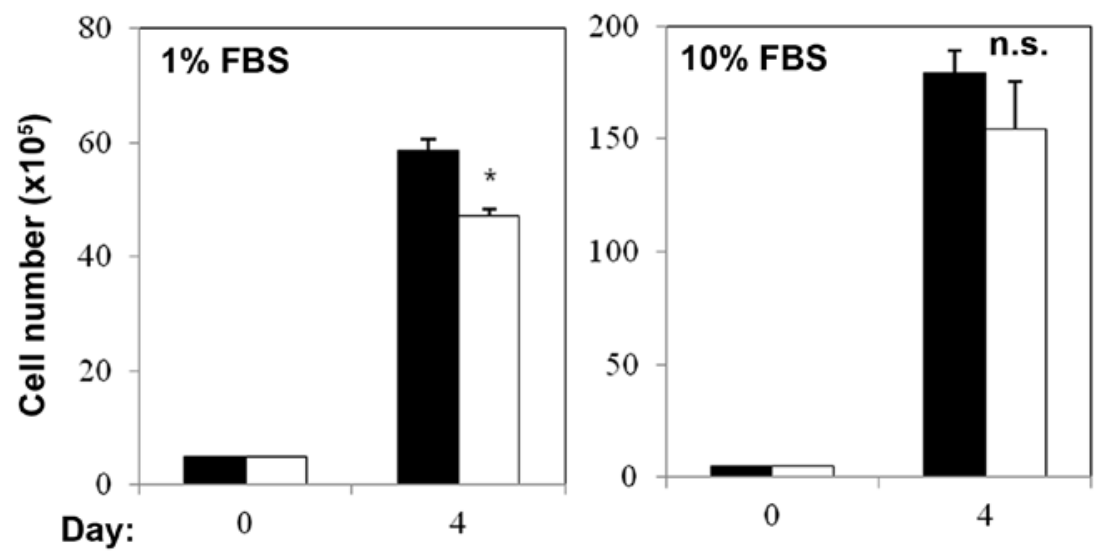

-Scramble

asiRNA

C

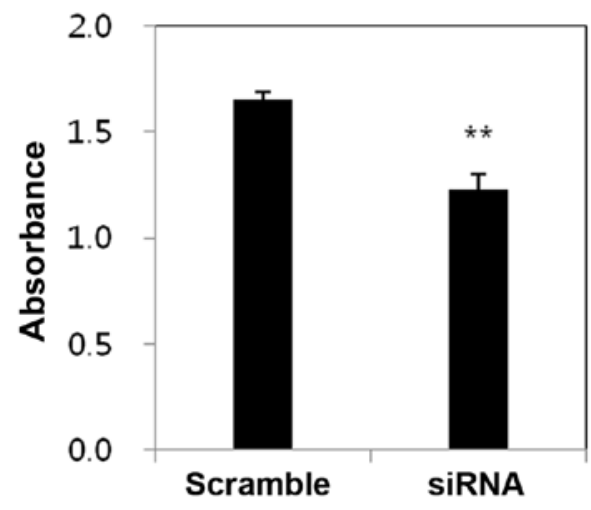

D

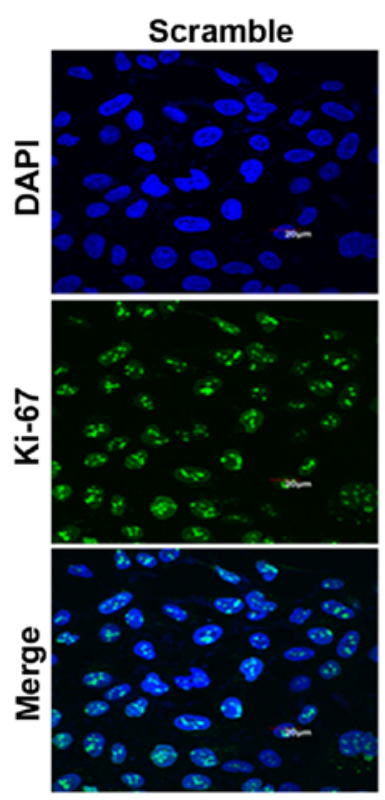

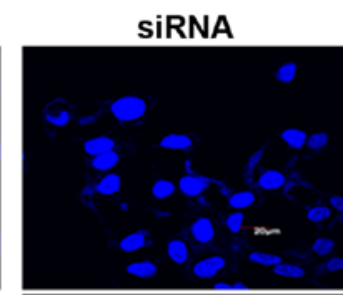
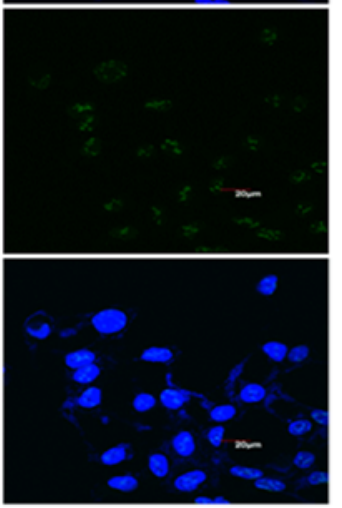

Figure 3. Decrease in cell growth and proliferation by silencing of NY-SAR-35 expression. HEK293/NY-SAR-35 cells were transfectected with either siRNA-control (scramble) or specific siRNA targeting NY-SAR-35 expression (siRNA). (A) NY-SAR-35 mRNA levels were evaluated by RT-PCR amplification. GAPDH mRNA expression was used as a control for equal gel loading in the bottom panel. (B) After transfection, the cells were grown in medium containing 1 or $10 \% \mathrm{FBS}$, and cell number was determined at day 4. (C) Cell proliferation was studied by measuring the incorporation of BrdU into synthesized DNA. (D) Immunofluorescence staining for DAPI stained nuclei blue and Ki-67-FITC (green) in the control scrambled or NY-SAR-35 siRNA treated HEK293/NY-SAR-35 cells were evaluated by confocal microscopy. Note the decrease in nuclear staining of Ki-67 in NY-SAR-35 specific siRNA treated cells (magnification, x600). Bars represent mean values, n.s., no significant difference; ${ }^{*} \mathrm{P}<0.05 ;{ }^{* *} \mathrm{P}<0.01$.

mitosis during spermatogenesis (Fig. 1D), we tested whether the NY-SAR-35 could regulate cell growth or proliferation. The increase in cell number of HEK293/NY-SAR-35 cells was determined by trypan blue exclusion assay. HEK293/ NY-SAR-35 cells grew on average 2.2 times more rapidly than HEK293 cells within the time period of 4 days (Fig. 2A).

Further examination of cell proliferation by BrdU assay showed an increase in DNA synthesis within three days of in vitro growth in HEK293/NY-SAR-35 cells (Fig. 2B).
Also, positive staining for Ki-67 (green, Fig. 2C) and PCNA (green, Fig. 2D), markers of cell proliferation, was found in HEK293/NY-SAR-35 cells. Ki-67 staining was restricted to the nucleus. In contrast, PCNA positivity showed staining intensity with both the nuclei and the cytoplasm. It was noted that NY-SAR-35 enhanced cell proliferation.

Furthermore, to confirm these effects of NY-SAR-35, knockdown of NY-SAR-35 was performed using RNAi molecule specific for NY-SAR-35. RT-PCR showed that 
A
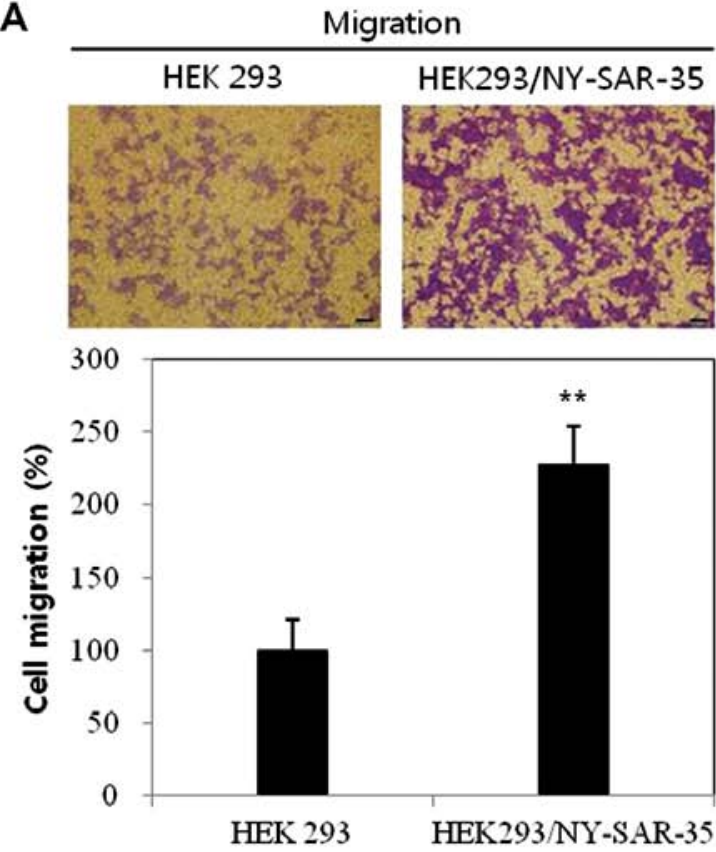

B
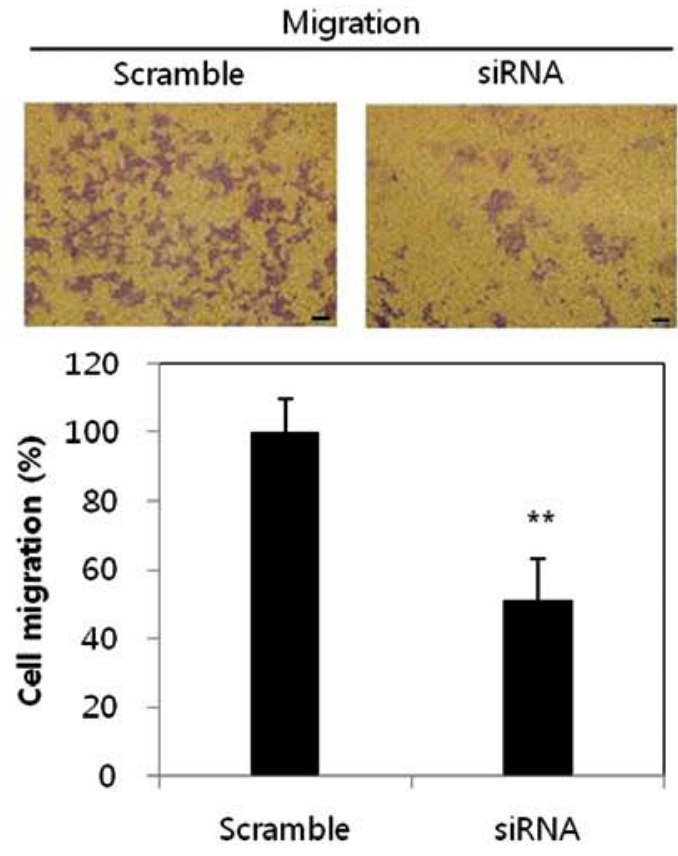
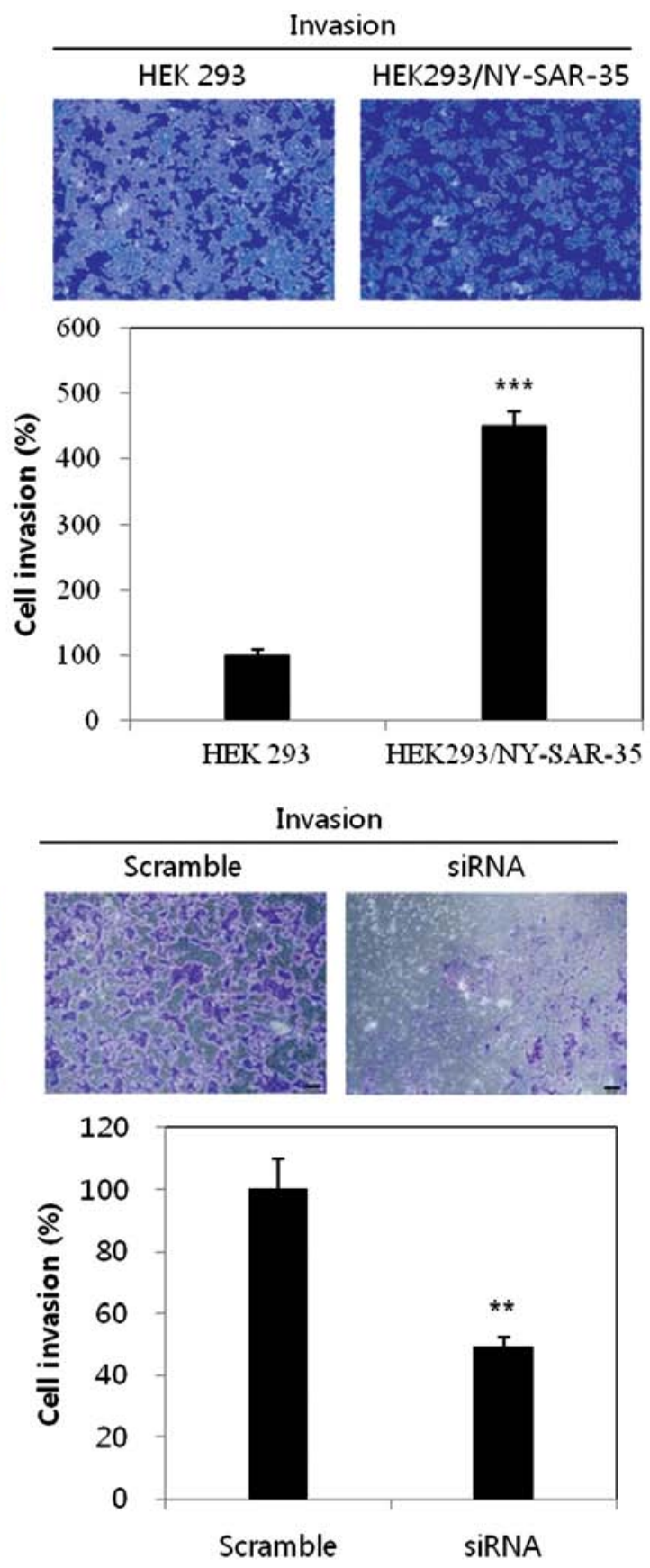

Figure 4. Enhancement of migration and invasion of HEK293 cells by expression of NY-SAR-35. (A) HEK293 and HEK293/NY-SAR-35 cells were subjected to the transwell migration assay and the Matrigel invasion assay. Data for relative migration and invasion activities of HEK293/NY-SAR-35 were expressed in percentage values compared with those of the control HEK293 cells (magnification, x100). (B) Transfected cells with either siRNA-control (scramble) or specific siRNA targeting NY-SAR-35 expression (siRNA) were also used in the transwell chamber assay and Matrigel-coated invasion assay. These cells were photographed (magnification, $\mathrm{x} 100$ ) and relative values for migration and invasion were expressed as a percentage of those cells. ${ }^{* *} \mathrm{P}<0.01 ;{ }^{* * *} \mathrm{P}<0.001$.

NY-SAR-35-siRNA effectively silenced NY-SAR-35 expression in HEK293/NY-SAR-35 cells (Fig. 3A). For growth assay, the control scrambled- and NY-SAR-35-siRNA treated cells were incubated for 4 days. The percentage of cell numbers dropped to $20 \%$ in NY-SAR-35 knocked-down cells compared to HEK293/NY-SAR-35 cells under media containing $1 \%$ FBS (Fig. 3B). Cell proliferation assay showed that DNA synthesis decreased in NY-SAR-35-silenced cells compared with HEK293/NY-SAR-35 cells (Fig. 3C). Immunofluorescent staining also showed that knocking down NY-SAR-35 expression significantly reduced positivity of Ki-67 (Fig. 3D).
NY-SAR-35 promotes cell migration and invasion in HEK293 cells. To understand the relationship between the expression of NY-SAR-35 and cellular motility, migration and invasion activity, we were examined in transwell assays using a Boyden chamber. HEK293/NY-SAR-35 cells showed that the migratory ability was increased to 2.3 -fold and invasive ability was also enhanced to 4.5-fold higher than the control HEK293 cells (Fig. 4A).

Furthermore, to confirm the migratory and invasive effects of NY-SAR-35 expression, NY-SAR-35 was knocked down in the HEK293/NY-SAR-35 cells and migration and invasion 
assays were performed. NY-SAR-35 silencing reduced migration and invasion of HEK293/NY-SAR-35 cells by $~ 50 \%$ (Fig. 4B). These findings indicated that NY-SAR-35 expression promoted cell migration and invasion in HEK293 cells.

\section{Discussion}

Many studies have analyzed CT antigens for their potential use in cancer immunotherapy because of their immunogenicity and restricted expression patterns $(1-4,10)$.

This study provided evidence that overexpression of NY-SAR-35 increased cell proliferation, migration, and invasion in HEK293 cells. We have for the first time demonstrated that NY-SAR-35 impacts cell proliferation, migration, and invasion. Although the role of NY-SAR-35 was not directly evaluated, these experiments illustrated the potential contribution of the CT antigen NY-SAR-35 in proliferative and migratory function.

During spermatogenesis, different CT antigens uniquely expressed in different germ cell types, demonstrating involvement in different phases of spermatogenesis $(3,12,26)$. Immunohistochemical staining in the testis tissue showed that NY-SAR-35 was mainly expressed in spermatogonia and primary and/or secondary spermatocytes, which was observed in most of CT antigens located on $\mathrm{X}$ chromosome (26). NY-SAR-35 was also present in the cytoplasmic compartment; however, predominant cytoplasmic expression was rare, with CT47, another CT antigen, as an example (27). This finding demonstrated NY-SAR-35 was associated with spermatogonia and spermatocyte and had possible roles during mitotic division of spermatogenesis.

We also found that the Ki-67 was localized within the nucleus of HEK293/NY-SAR-35 cells. The Ki-67 antigen has been detected in proliferating cells in all phases except G0 phase of the cell-division cycle of normal and neoplastic cells. Equal to Ki-67, PCNA detection was increased in HEK293/ NY-SAR-35 cells. Possibly NY-SAR-35 was able to perform a number of functions within proliferating cells.

In addition, the functional study of NY-SAR-35 in HEK293 cell lines demonstrated that HEK293/NY-SAR-35 cells have greater migratory and invasive abilities compared to control cells; whereas suppression of NY-SAR-35 showed reduction in migration and invasion capabilities of HEK293/NY-SAR-35 cells.

The regulations of NY-SAR-35-mediated cell proliferation and migration were determined, however, various questions need to be addressed and investigated in further studies, including: what are the potential mechanisms to increase the cell proliferation and migration of HEK293 cells by expression of NY-SAR-35? How do the patterns of gene expression change in HEK293 cells by overexpression of NY-SAR-35? Also it is important to explore whether the phenotypic changes are shown in cancer cell types by overexpression of NY-SAR-35.

In conclusion, the above data demonstrated that the expression of NY-SAR-35 stimulated the cell growth and proliferation in vitro assay. Also, NY-SAR-35 enhanced cell migration and invasion, suggesting that NY-SAR-35 gene appears to be able to mediate proliferative and migratory signals. In addition, NY-SAR-35 may be a useful tool in research on the linkage between embryology and tumor biology.

\section{Acknowledgements}

This study was supported by Basic Science Research Program through the National Research Foundation of Korea (NRF) funded by the Ministry of Education(NRF-2012R1A1A2041573).

\section{References}

1. Scanlan MJ, Gure AO, Jungbluth AA, Old LJ and Chen YT: Cancer/testis antigens: An expanding family of targets for cancer immunotherapy. Immunol Rev 188: 22-32, 2002.

2. Scanlan MJ, Simpson AJ and Old LJ: The cancer/testis genes: Review, standardization, and commentary. Cancer Immun 4: 1, 2004.

3. Simpson AJ, Caballero OL, Jungbluth A, Chen YT and Old LJ: Cancer/testis antigens, gametogenesis and cancer. Nat Rev Cancer 5: 615-625, 2005.

4. Zendman AJ, Ruiter DJ and Van Muijen GN: Cancer/testisassociated genes: Identification, expression profile, and putative function. J Cell Physiol 194: 272-288, 2003.

5. Chen YT, Scanlan MJ, Sahin U, Türeci O, Gure AO, Tsang S, Williamson B, Stockert E, Pfreundschuh $M$ and Old LJ: A testicular antigen aberrantly expressed in human cancers detected by autologous antibody screening. Proc Natl Acad Sci USA 94: 1914-1918, 1997.

6. Song MH, Choi KU, Shin DH, Lee CH and Lee SY: Identification of the cancer/testis antigens AKAP3 and CTp11 by SEREX in hepatocellular carcinoma. Oncol Rep 28: 1792-1798, 2012.

7. Chen YT, Chadburn A, Lee P, Hsu M, Ritter E, Chiu A, Gnjatic S, Pfreundschuh M, Knowles DM and Old LJ: Expression of cancer testis antigen CT45 in classical Hodgkin lymphoma and other B-cell lymphomas. Proc Natl Acad Sci USA 107: 3093-3098, 2010.

8. Song MH,Ha JM, Shin DH, Lee CH, Old L and Lee SY: KP-CoT-23 (CCDC83) is a novel immunogenic cancer/testis antigen in colon cancer. Int J Oncol 41: 1820-1826, 2012.

9. Song MH, Ha JC, Lee SM, Park YM and Lee SY: Identification of BCP-20 (FBXO39) as a cancer/testis antigen from colon cancer patients by SEREX. Biochem Biophys Res Commun 408: 195-201, 2011.

10. Fratta E, Coral S, Covre A, Parisi G, Colizzi F, Danielli R, Nicolay HJ, Sigalotti L and Maio M: The biology of cancer testis antigens: Putative function, regulation and therapeutic potential. Mol Oncol 5: 164-182, 2011.

11. Ong SE and Mann M: A practical recipe for stable isotope labeling by amino acids in cell culture (SILAC). Nat Protoc 1: 2650-2660, 2006.

12. Dobrynin P, Matyunina E, Malov SV and Kozlov AP: The novelty of human cancer/testis antigen encoding genes in evolution. Int J Genomics 2013: 105108, 2013.

13. Doyle JM, Gao J, Wang J, Yang M and Potts PR: MAGE-RING protein complexes comprise a family of E3 ubiquitin ligases. Mol Cell 39: 963-974, 2010.

14. Epping MT, Wang L, Edel MJ, Carlée L, Hernandez M and Bernards R: The human tumor antigen PRAME is a dominant repressor of retinoic acid receptor signaling. Cell 122: 835-847, 2005.

15. Whitehurst AW, Xie Y, Purinton SC, Cappell KM, Swanik JT, Larson B, Girard L, Schorge JO and White MA: Tumor antigen acrosin binding protein normalizes mitotic spindle function to promote cancer cell proliferation. Cancer Res 70: 7652-7661, 2010.

16. Cappell KM, Sinnott R, Taus P, Maxfield K, Scarbrough M and Whitehurst AW: Multiple cancer testis antigens function to support tumor cell mitotic fidelity. Mol Cell Biol 32: 4131-4140, 2012.

17. Shang B, Gao A, Pan Y, Zhang G, Tu J, Zhou Y, Yang P, Cao Z, Wei Q, Ding Y, et al: CT45A1 acts as a new proto-oncogene to trigger tumorigenesis and cancer metastasis. Cell Death Dis 5: e1285, 2014.

18. Bai S, He B and Wilson EM: Melanoma antigen gene protein MAGE-11 regulates androgen receptor function by modulating the interdomain interaction. Mol Cell Biol 25: 1238-1257, 2005.

19. Monte M, Simonatto M, Peche LY, Bublik DR, Gobessi S, Pierotti MA, Rodolfo M and Schneider C: MAGE-A tumor antigens target p53 transactivation function through histone deacetylase recruitment and confer resistance to chemotherapeutic agents. Proc Natl Acad Sci USA 103: 11160-11165, 2006. 
20. Cilensek ZM, Yehiely F, Kular RK and Deiss LP: A member of the GAGE family of tumor antigens is an anti-apoptotic gene that confers resistance to Fas/CD95/APO-1, Interferon-gamma, taxol and gamma-irradiation. Cancer Biol Ther 1: 380-387, 2002.

21. Hamer G, Gell K, Kouznetsova A, Novak I, Benavente R and Höög C: Characterization of a novel meiosis-specific protein within the central element of the synaptonemal complex. J Cell Sci 119: 4025-4032, 2006.

22. D'Arcy P, Maruwge W, Wolahan B, Ma L and Brodin B: Oncogenic functions of the cancer-testis antigen SSX on the proliferation, survival, and signaling pathways of cancer cells. PLoS One 9: e95136, 2014.

23. Lee SY, Obata Y, Yoshida M, Stockert E, Williamson B, Jungbluth AA, Chen YT, Old LJ and Scanlan MJ: Immunomic analysis of human sarcoma. Proc Natl Acad Sci USA 100: 2651-2656, 2003.
24. Kim YD, Park HR, Song MH, Shin DH, Lee CH, Lee MK and Lee SY: Pattern of cancer/testis antigen expression in lung cancer patients. Int J Mol Med 29: 656-662, 2012.

25. Park JH, Song MH, Lee CH, Lee MK, Park YM, Old L and Lee SY: Expression of the human cancer/testis antigen NY-SAR-35 is activated by $\mathrm{CpG}$ island hypomethylation. Biotechnol Lett 33: 1085-1091, 2011

26. Cheng YH, Wong EW and Cheng CY: Cancer/testis (CT) antigens, carcinogenesis and spermatogenesis. Spermatogenesis 1: 209-220, 2011

27. Caballero OL and Chen YT: Cancer/testis (CT) antigens: Potential targets for immunotherapy. Cancer Sci 100: 2014-2021, 2009. 